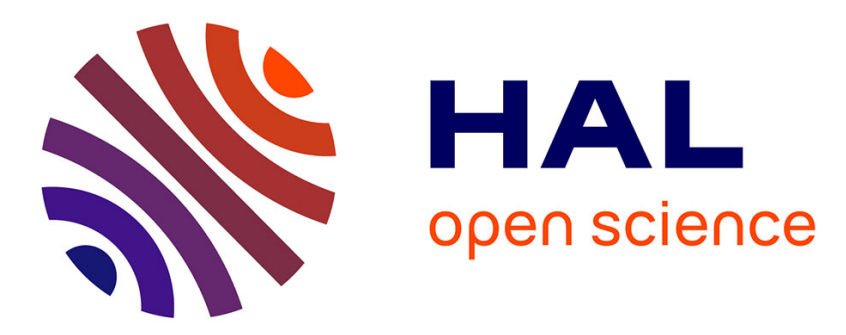

\title{
Intelligent Antenna Selection Decision in IEEE 802.15.4 Wireless Sensor Networks: An Experimental Analysis
}

Husain Rehmani Mubashir, Abderrezak Rachedi, Stéphane Lohier, Thierry Alves, Benoit Poussot

\section{- To cite this version:}

Husain Rehmani Mubashir, Abderrezak Rachedi, Stéphane Lohier, Thierry Alves, Benoit Poussot. Intelligent Antenna Selection Decision in IEEE 802.15.4 Wireless Sensor Networks: An Experimental Analysis. Computers and Electrical Engineering, 2014, 40 (2), pp.443-455. 10.1016/j.compeleceng.2013.11.021 . hal-00879360

\section{HAL Id: hal-00879360 https://hal.science/hal-00879360}

Submitted on 15 Nov 2013

HAL is a multi-disciplinary open access archive for the deposit and dissemination of scientific research documents, whether they are published or not. The documents may come from teaching and research institutions in France or abroad, or from public or private research centers.
L'archive ouverte pluridisciplinaire HAL, est destinée au dépôt et à la diffusion de documents scientifiques de niveau recherche, publiés ou non, émanant des établissements d'enseignement et de recherche français ou étrangers, des laboratoires publics ou privés. 


\title{
Intelligent Antenna Selection Decision in IEEE 802.15.4 Wireless Sensor Networks: An Experimental Analysis
}

\author{
Mubashir Husain Rehmani ${ }^{1 \mathrm{a}, \mathrm{c}}$, Abderrezak Rachedia ${ }^{\mathrm{a}}$, Stéphane Lohier ${ }^{\mathrm{a}}$, \\ Thierry Alves ${ }^{\mathrm{b}}$, Benoit Poussot ${ }^{\mathrm{b}}$ \\ ${ }^{a}$ Université Paris-Est Marne-la-Vallée, LIGM, PasNet, France \\ ${ }^{b}$ Université Paris-Est Marne-la-Vallée, ESYCOM, France \\ ${ }^{c}$ COMSATS Institute of Information Technology, Wah Cantt, Pakistan
}

\begin{abstract}
The goal of this paper is to study the feasibility of making intelligent antenna selection decision in IEEE 802.15.4 Wireless Sensor Networks (WSNs). This study provides us the basis to design and implement software defined intelligent antenna switching capability to wireless sensor nodes based on Received Signal Strength Indicator (RSSI) link quality metric. First, we discuss the results of our newly designed radio module (Inverted-F Antenna) for $2.4 \mathrm{GHz}$ bandwidth (WSNs). Second, we propose an intelligent antenna selection strategy to exploit antenna diversity. Third, we propose the prototype of our diversity antenna for the TelosB mote and the intelligent switch design. Finally, we compare the performance of the built-in TelosB antenna with our proposed external antenna in both laboratory and realistic environments. Experimental results confirm the gain of 6 to $10 \mathrm{~dB}$ of the proposed radio module over the built-in radio module of the TelosB motes.
\end{abstract}

Keywords:

Wireless sensor networks, IEEE 802.15.4, ZigBee, RSSI, Antenna diversity

\footnotetext{
${ }^{1}$ Corresponding Author Address:

Laboratoire d'Informatique Gaspard Monge (LIGM), PasNet,

Université Paris-Est Marne-la-Vallée,

5, Bd Descartes,

77454, Marne-la-vallée Cedex 2, France.

Phone: +33 (0) 160957737

Fax: +33 (0) 160957551
} 


\section{Introduction}

In our daily life, Wireless Sensor Networks (WSNs) can be seen everywhere. The applications of these WSNs ranges from environmental monitoring like wildlife tracking, habitat monitoring, forest fire detection, mine safety monitoring to military applications like target detection, and tracking $[1,2]$. However, the performance of these WSNs depends upon the quality of the wireless link, the built-in antenna available on the sensor device, and the antenna diversity. In WSNs, there are several application scenarios where a clear line-of-sight (LOS) between the sender and receiver is not present $[3,4,5,6]$. This results in fading of the signal and causes multipath propagation. Furthermore, the signal between transmitter-receiver pair is prone to attenuation and distortion, resulting in packet losses. The main reason of attenuation and distortion is due to path loss, multipath fading, radiation characteristics, antenna orientation, and Doppler effects and are highly dependent upon the location and surrounding of the transmitter-receiver pair [8]. Antenna diversity is a way in which two antennas are attached with the sensor node to improve the quality and reliability of the wireless link [6], [7].

In IEEE 802.15.4, the quality of the link is measured by two metrics: Link Quality Indicator (LQI) and Received Signal Strength Indicator (RSSI). These two metrics are offered by the IEEE 802.15.4 physical layer, which can then be used by the routing layer to select good quality routes. For instance, the impact of LQI-Based routing metrics on the performance of a One-to-One routing protocol for IEEE 802.15.4 Multihop Networks has been studied by the authors in [9]. The authors in [10] provided a detailed study on the comparison of LQI and RSSI metrics. However, aforementioned works $[9,10]$ did not consider the antenna diversity. Recently, the authors in [11] showed that significant gain can be achieved through antenna diversity but their work mainly focuses on directional antennas for smart-phone like mobile devices. In [12], the authors focus on target coverage problem using directional sensor and antennas.

Based on link quality metric RSSI, our ultimate goal is to design and imple- 
ment software defined intelligent antenna switching capability to wireless sensor nodes. More precisely, we want to attach an external antenna with the sensor nodes besides the built-in antenna to achieve antenna diversity. Then, based on wireless link condition, the sensor node switch to the appropriate antenna for communication.

Our Contributions: This paper makes the following contributions:

- As a first step, we design a new radio module, an Inverted-F Antenna for $2.4 \mathrm{GHz}$ bandwidth WSNs. In fact, we study the feasibility of making intelligent antenna selection decision in IEEE 802.15.4 WSNs. This study will provide us the basis to design and implement software defined intelligent antenna switching capability to wireless sensor nodes based on link quality metric, such as RSSI.

- We perform extensive experiments in outdoor garden environment, indoor office environment, reverberation chamber and anechoic chamber. We then compare results of our antenna with the built-in TelosB antenna. Experimental results confirm the effectiveness of the proposed radio module over the built-in radio module of the TelosB motes.

- In the second step, we propose an intelligent antenna selection strategy to exploit antenna diversity. In this strategy, wireless sensor nodes predict the values of RSSI of built-in and external antenna and make the antenna selection decision adaptively. In fact, the proposed antenna selection strategy is based on Exponentially Weighted Moving Average (EWMA) and rely on the historical observations of the RSSI values of built-in and external antenna.

- We then propose the prototype of our diversity antenna for the TelosB mote and the intelligent switch design.

- Finally, we discuss issues and challenges related to intelligent antenna selection and antenna diversity in the context of WSNs, which open new research directions. 
Organization of the paper: The remainder of this paper is organized as follows: In section 2, we discuss Inverted-F antenna design. Performance analysis of built-in and external antenna is done in section 3. We then propose EWMA based intelligent antenna selection strategy in section 4. Open issues and challenges are discussed in section 5. Section 6 discusses the related work. Finally, section 7 concludes the paper with future research directions.

\section{Inverted-F Antenna (IFA) Design}

We now describe the design and connection procedure of our antenna [13, 14] with the TelosB mote, which is widely used in WSNs research. According to the TelosB datasheet [15], we have implemented an SubMiniature version A (SMA) connector in order to connect our small external antenna. This antenna is called as Inverted-F Antenna (IFA). Fig. 1 shows Inverted-F antenna attached with TelosB mote. Compared to the embedded antenna, this one is different in the sense that it couples to its one ground plane. This particularity makes the antenna working on Ultra Wide Band (UWB) from 2070 to $3140 \mathrm{MHz}$ for a -10 $\mathrm{dB}$ matching bandwidth. The current excited by the IFA on its ground plane make the radiation pattern to have a null on the Y-axis. The gain is almost constant in the $\mathrm{XZ}$ plane and is around $1 \mathrm{dBi}$ (cf. Fig. 2 for the design and radiation pattern). This antenna has two advantages :

-Firstly, the matching bandwidth is much more important from the embedded antenna. Making it a little less sensitive to proximity effects.

-Secondly, as the gain is almost constant in the ZY plane and have a null in the OX direction, thus limiting troubles from the USB connector.

\section{Performance Analysis of Built-in and External Antenna}

We first describe the methodology in this section and then proceed with the performance analysis. 


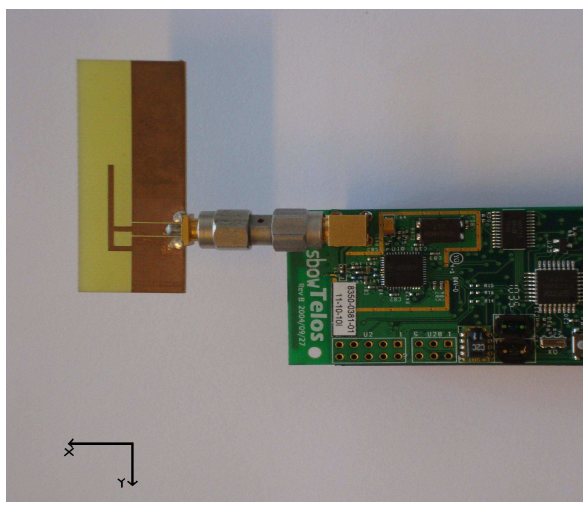

Figure 1: Inverted-F antenna attached with TelosB mote.

Methodology: Since our goal is to consider RSSI in making the antenna selection decision, we first perform extensive experiments to study the behaviour of RSSI in four different environments, namely, (1) Indoor Office Environment, (2) Outdoor Garden Environment, (3) Reverberation chamber, and (4) Anechoic chamber. Note that the reverberation chamber is used to simulate indoor environment with multipath and the anechoic chamber is used to simulate outdoor environment.

Implementation Setup: In this section, we describe our implementation setup. We consider TelosB motes [16], which are equipped with Chipcon CC2420 radio module [17]. The radio module $\mathrm{CC} 2420$ in its new versions is used in many radio devices. The transceiver of TelosB motes operate in $2.4 \mathrm{GHz}$ band. We use RSSI as a link quality metric to study the performance of our newly designed antenna. The RSSI provides the signal strength at the receiver (in $\mathrm{dBm}$ ) [10]. The CC2420 calculates the RSSI over 8 symbol periods and stores the result in its RSSI.RSSI_VAL register. Chipcon specifies the following formula to compute the received signal power $(\mathrm{P})$ in $\mathrm{dBm}: \mathrm{P}=$ RSSI VAL + RSSI OFFSET, where RSSI OFFSET is equal to -45 . We refer to this power, $\mathrm{P}$ (in $\mathrm{dBm}$ ) as RSSI throughout this paper.

We consider two types of TelosB motes in our experiment: (1) sink node, which is connected with the serial port of the Laptop, and (2) the sending mote, 

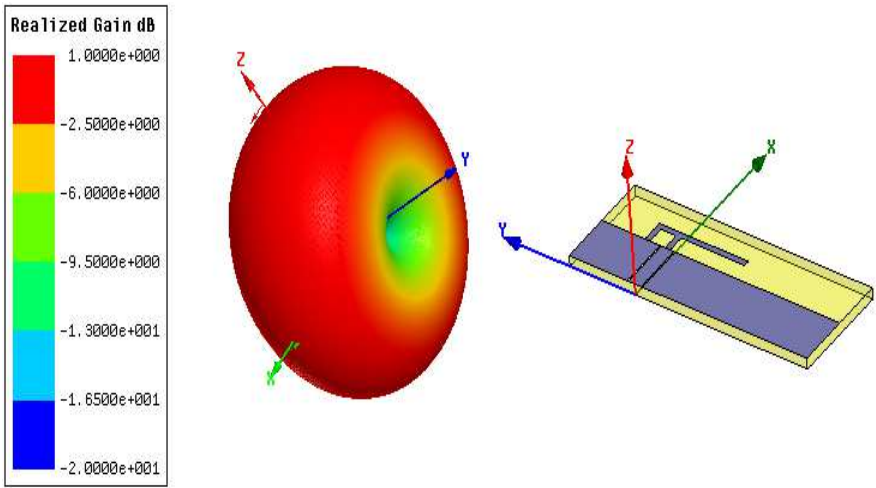

Figure 2: Realized Gain (dB) and Design of Inverted-F Antenna for $2.4 \mathrm{GHz} \mathrm{WSNs}$.

which sends packet with a time delay of $500 \mathrm{~ms}$. Total 100 packets were sent by the sending mote. As soon as the sink node receives packet by the sending mote, it saves the RSSI value of each corresponding packet. The sink node and the sender node is placed 1 feet and 1 meter apart. We carried out our experiment at 3 different power levels: $0 \mathrm{dBm},-10 \mathrm{dBm}$ and $-25 \mathrm{dBm}$. To change the transmission power, we change the default transmission power of CC2420 i.e., 0 dBm (CC2420_DEF_RFPOWER = 31), to $-10 \mathrm{dBm}$ and $-25 \mathrm{dBm}$, by changing the register values to 11 and 3, respectively. In order to study the impact of LOS and multipath on RSSI for both antennas, we place the sender node at five different locations, while the receiving node's position was fixed (cf. Fig. 5). We now describe the results for each environment.

\subsection{Indoor Office Environment}

We first consider indoor office environment in which we have walls, computers, tables, and other reflecting material. Moreover, we also have human activity and the experiments were conducted in the presence of a single person. Fig. 3 shows the plan for the indoor office environment of Universite Paris Est, Copernice Building, 4th floor, where we conducted our experiments.

Fig. 4 compares the RSSI values of built-in antenna and external IFA antenna for each received packet with $0 \mathrm{dbm},-10 \mathrm{dBm}$ and $-25 \mathrm{dBm}$ power levels. Results 


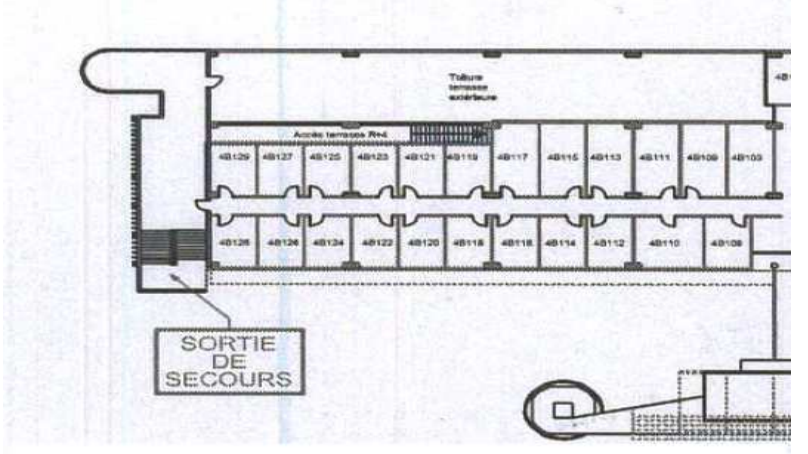

Universite Paris Est, Copernic Building, 4th Floor

Figure 3: Indoor offices plan of Universite Paris Est, Copernic Building, 4th floor.

in Fig. 4(a), 4(b) and 4(c) were taken when the distance between the sender node and the receiving node was 1 feet. While Fig. 4(d) and 4(e) shows the results when the sender node and the receiving node were 1 meter apart. Note that in Fig. 4(d) and 4(e), the sender node is placed at five different locations (cf. Fig. 5).

The IFA antenna provides higher power levels i.e., RSSI values at the receiver. More precisely, when power level is $0 \mathrm{dBm}$, the external IFA antenna provides $-33.81 \mathrm{dBm}$ power compared to $-58.4 \mathrm{dBm}$ power for the built-in antenna, i.e., the gain of $25 \mathrm{~dB}$. When power level is $-10 \mathrm{dBm}$, the external IFA antenna provides $-44.41 \mathrm{dBm}$ power compared to $-74.0 \mathrm{dBm}$ power for the builtin antenna, i.e., the gain of $30 \mathrm{~dB}$. And when the power level is $-25 \mathrm{dBm}$, the external IFA antenna provides $-58.95 \mathrm{dBm}$ power compared to $-76.67 \mathrm{dBm}$ power for the built-in antenna, i.e., the gain of around $17.75 \mathrm{~dB}$. As mentioned earlier, to study the affect of LOS and multipath, we consider five different positions for the sender node. In addition, we also increase the distance between the sender node and receiving node to 1 meter. We now observe the RSSI values for the external and built-in antenna in Fig. 4(d) and 4(e). As can be seen in the figure, the RSSI value is different for every position (cf. Fig. 5). This is due to the multipath affect and due to the absence of clear line of sight between the sender and the receiver nodes. In summary, results in Fig. 4(a), 4(b) and 4(c) confirm 


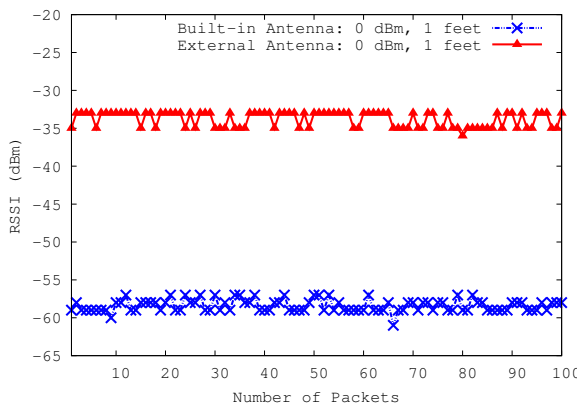

(a)

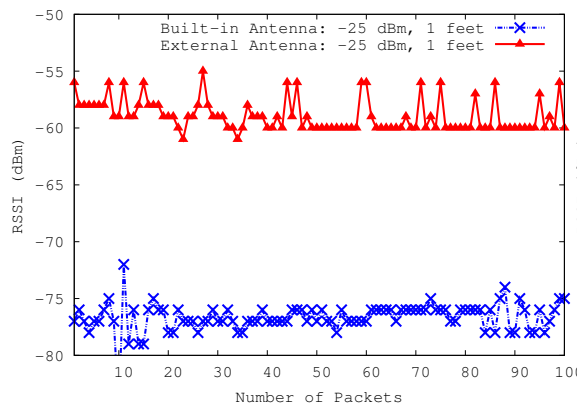

(c)

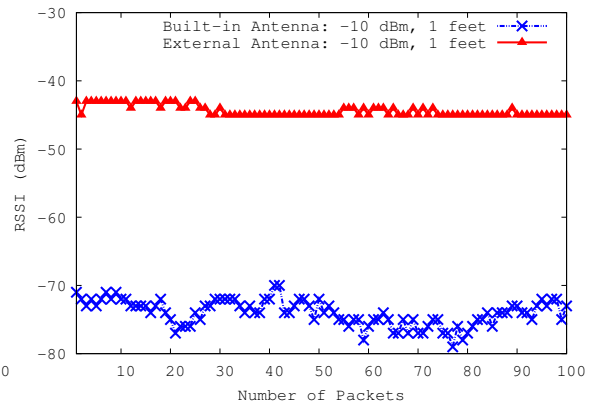

(b)

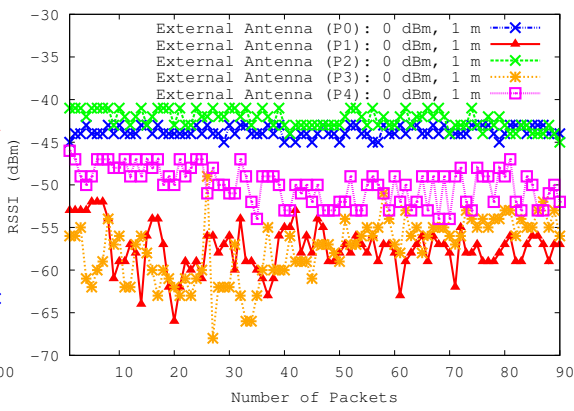

(d)

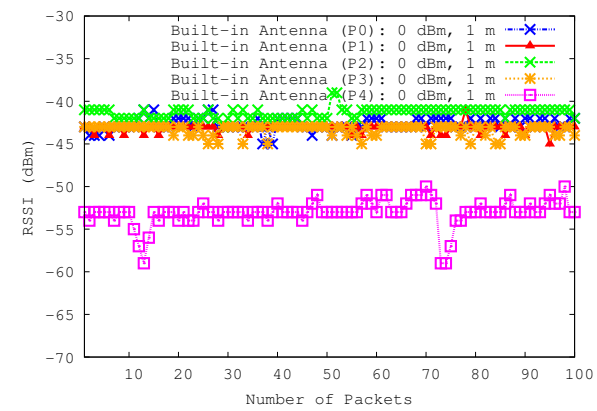

(e)

Figure 4: Number of Packets and RSSI in Indoor office environment. (a) 0 dBm Power. (b) $-10 \mathrm{dBm}$ Power. (c) $-25 \mathrm{dBm}$ Power. (d) External antenna placed at five different locations with $0 \mathrm{dBm}$ Power. (e) Built-in antenna placed at five different locations with $0 \mathrm{dBm}$ Power. 


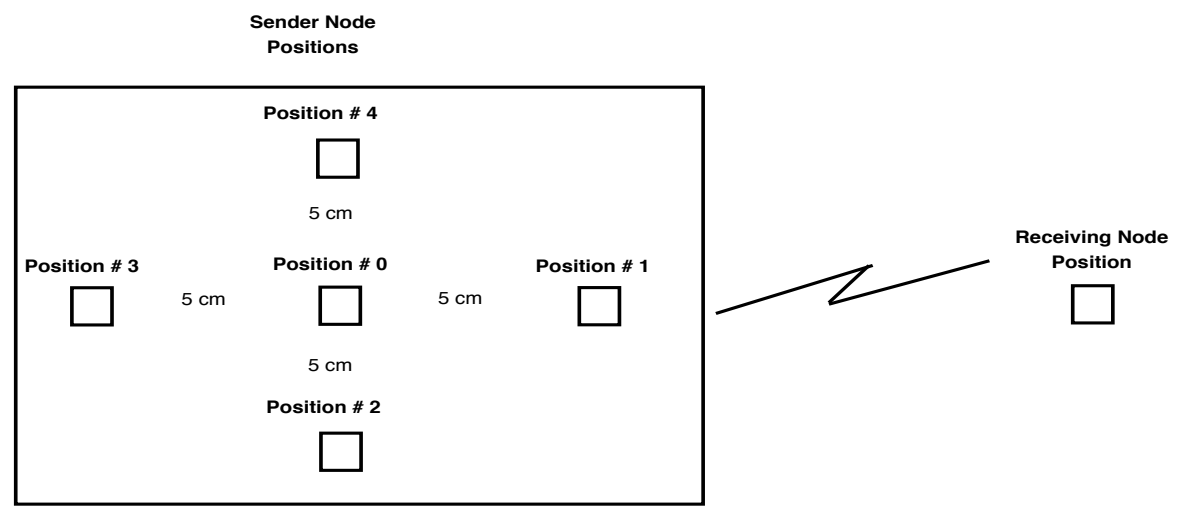

Figure 5: Sender and Receiving nodes positions.

that the newly designed IFA antenna can provide good link reliability, suitable for wireless radio communication in IEEE 802.15.4 based WSNs.

We also conclude that when the sender and receiver nodes are at short distance, we observe the different RSSI values for the two antennas (cf. Fig. 4(a), 4(b) and $4(\mathrm{c})$ ). On the other hand, when the sender and receiver nodes are at long distance, the RSSI values received at both the antennas fluctuates (cf. Fig. 4(d) and 4(e)). This is because of multipath fading and scattering effects, which ultimately changes the polarization of waves.

\subsection{Reverberation Chamber}

Reverberation chambers are used to study the effects of multi-path propagation environments [26]. Fig. 6 and Fig. 7 shows the outside and inside view of Reverberation chamber that we considered in our experiments. We use Model 2005 Azimuth Stirrer Command Set with the speed of $2 \mathrm{rpm}$ to create highly dynamic environment in the reverberation chamber by rotating it.

We compare the RSSI values of built-in antenna and external IFA antenna in Reverberation Chamber, for each received packet with $-25 \mathrm{dBm}$ power level (cf. Fig. 8). More precisely, results in Fig. 8 were taken when the nodes were 1 meter apart and the sender node is placed at two different locations (cf. Fig. 5).

It can be clearly seen in Fig. 8 that RSSI values in Reverberation chamber highly fluctuates. This is due to the highly dynamic environment (Azimuth 


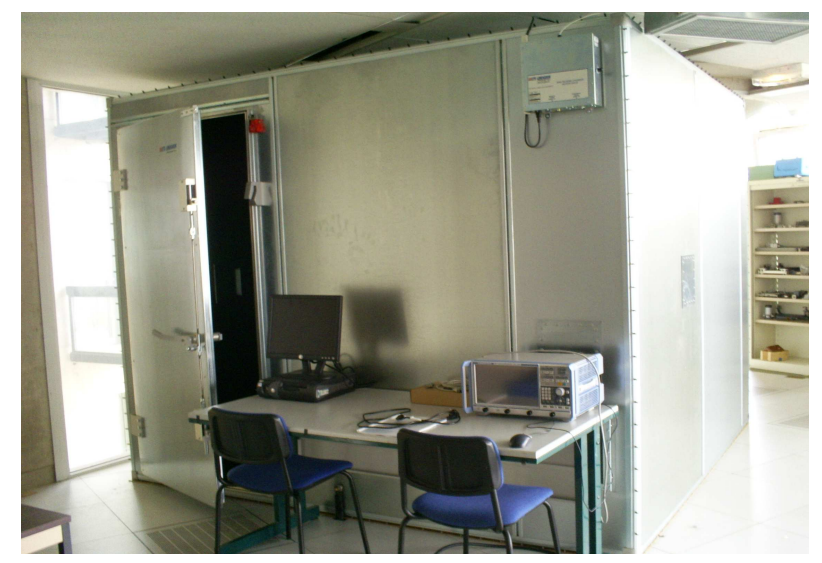

Figure 6: Outside view of the Reverberation Chamber in Université Paris-Est Marne-la-Vallée, LIGM, PasNet, France.

Stirrer rotation at $2 \mathrm{rpm}$ ). This results in multi-path and thus we have varying RSSI values for both antennas. Due to this multi-path phenomenon, we calculate the delivery ratio of the packet for both antennas at 5 distinct positions. Another important point that need to be noted over here is that the RSSI value for both antennas is time varying, means, for any time instant $t$, external antenna is performing good, while for time instant $t+1$, built-in antenna gives good performance. Thus, we plan to use this spatio-temporal variation in RSSI to select appropriate antenna (cf. Section 4 for more details).

Fig. 9 shows the delivery ratio of both antennas at five different positions. The results attest the obtained low and varying delivery ratios are mainly due to the highly dynamic environment in the reverberation chamber. However, we are unable to make any conclusion that which antenna is performing better in reverberation chamber.

Thus, in order to well understand the gain in reverberation chamber, we perform an statistical analysis of the received power for both the built-in and external antenna. The graphical representation in Fig. 10 permits to know the probability of the received power level during the measurement campaign. For the same probability, the received signal can be higher or lower according to the antenna and this is much more useful to compare the antennas than by 


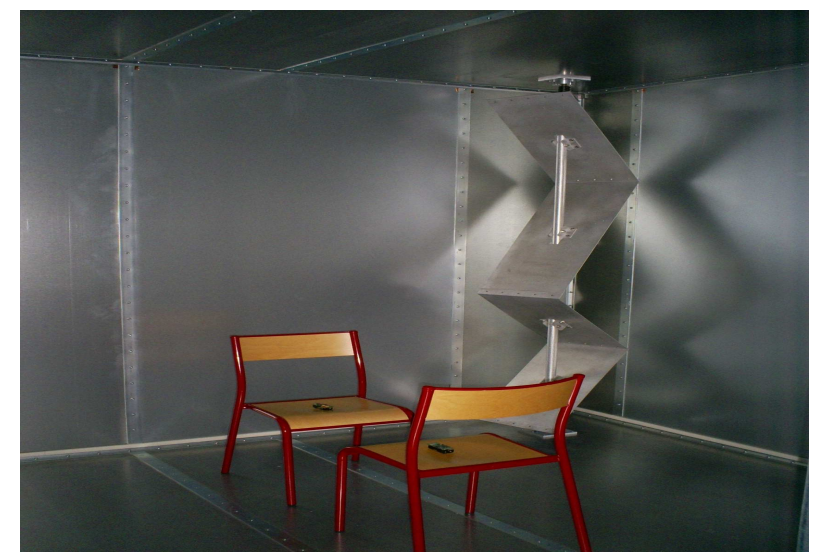

Figure 7: Inside view of the Reverberation Chamber in Université Paris-Est Marne-la-Vallée, LIGM, PasNet, France.

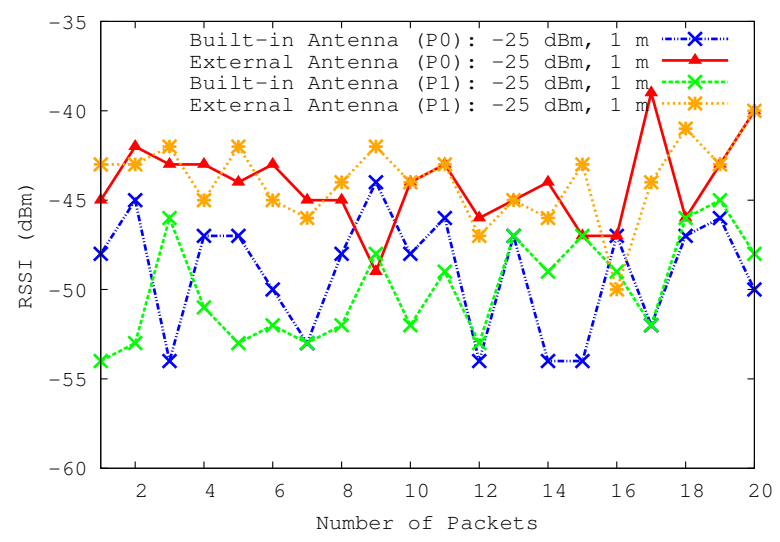

Figure 8: Number of Packets and RSSI in Reverberation chamber environment.

just looking at the time domain received power. We plot Empirical Cumulative Distribution Function (ECDF) on the log scale [42] that we obtained in the reverberation chamber. Fig. 10 shows the empirical cumulative distribution function for the two antennas. At $10 \%$ of reliability, Fig. 10 shows the gain of 6 to $8 \mathrm{~dB}$ improvement with external antenna in all the cases. Note that in Fig. 10(c), we have very less gain i.e., $3.5 \mathrm{~dB}$ gain and this is because we did not receive enough packets to calculate the gain. 


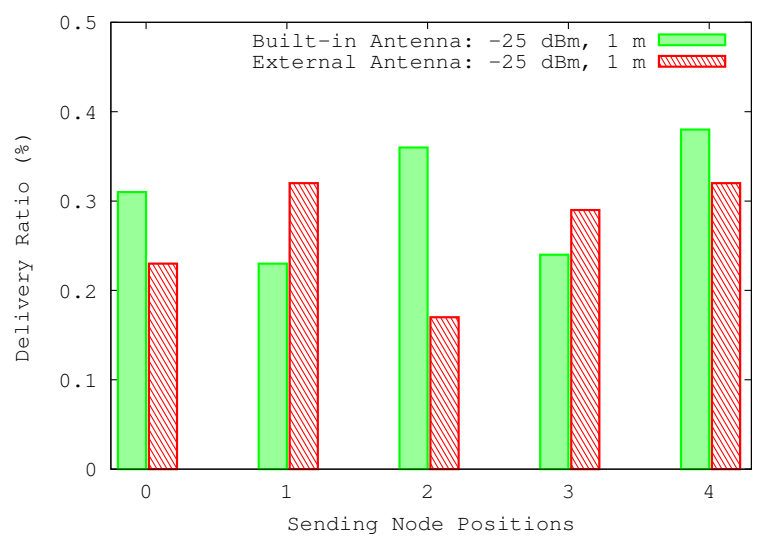

Figure 9: Delivery ratio of Built-in and External antenna in Reverberation chamber environment.

\subsection{RF Anechoic Chamber}

RF Anechoic chambers are designed to resemble a near free space environment in such a way that no outside interference can enter inside the chambers. Moreover, there is no reflection of electromagnetic waves inside the chamber.

In Fig. 11, we compare the RSSI values of built-in antenna and external IFA antenna in Anechoic Chamber, for each received packet with $0 \mathrm{dbm},-10 \mathrm{dBm}$ and $-25 \mathrm{dBm}$ power levels. Results in Fig. 11(a), 11(b) and 11(c) were taken when the nodes were placed 1 meter apart, while Fig. 11(d) and 11(e) shows the results when the nodes were 1 meter apart and the sender node is placed at five different locations (cf. Fig. 5).

Figure 11 witness that the external antenna outperforms the built-in antenna of TelosB mote. More precisely, with $0 \mathrm{dBm}$ power (cf. Fig. 11(a)), the RSSI of external antenna is $-45.51 \mathrm{dBm}$ and the RSSI of built-in antenna is $-53.94 \mathrm{dBm}$ i.e., the gain of $8.5 \mathrm{~dB}$. Similarly, with $-10 \mathrm{dBm}$ power (Fig. 11(b)), the RSSI of external antenna is $-54.96 \mathrm{dBm}$ and the RSSI of built-in antenna is $-64.3 \mathrm{dBm}$ i.e., the gain of $9.3 \mathrm{~dB}$. The same behaviour can be observed in Fig. 11(c), when the power is $-25 \mathrm{dBm}$. 


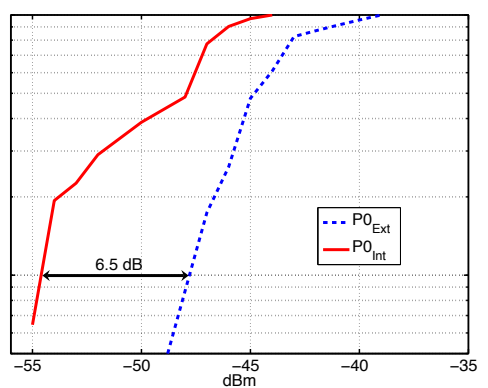

(a)

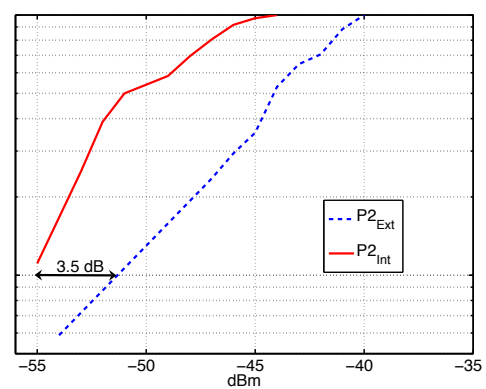

(c)

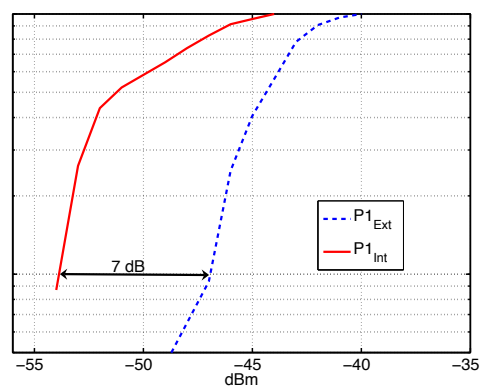

(b)

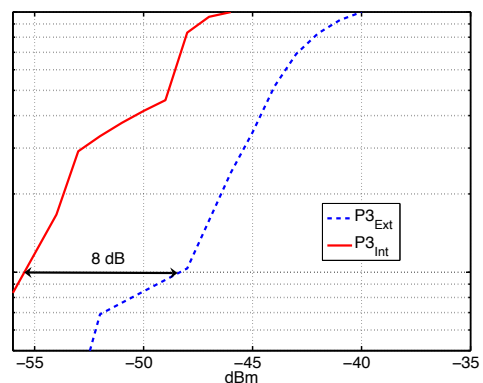

(d)

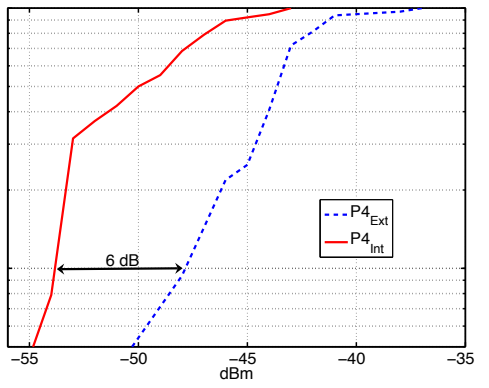

(e)

Figure 10: Emperical Cumulative Distributed Function for External and Built-in Antenna in the Reverberation Chamber (a) position 0 (b) position 1 (c) position 2 (d) position 3 (e) position 4 


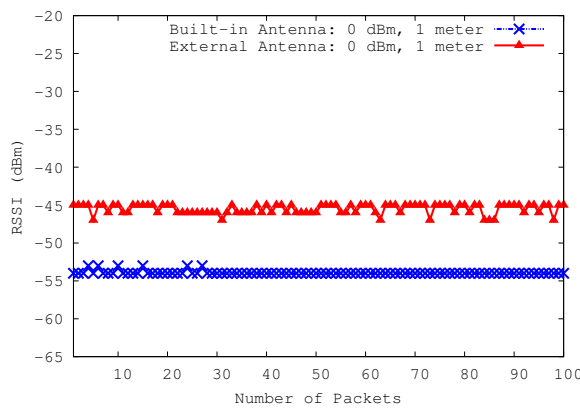

(a)

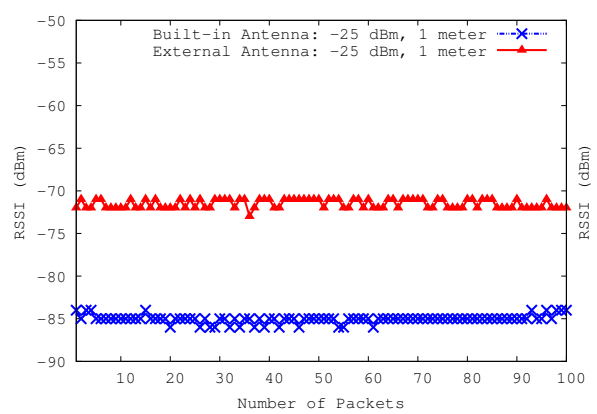

(c)

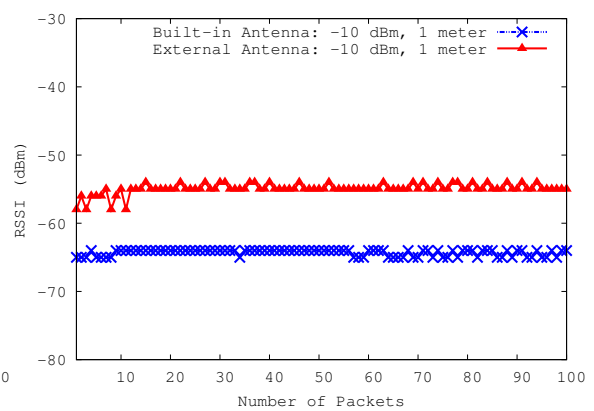

(b)

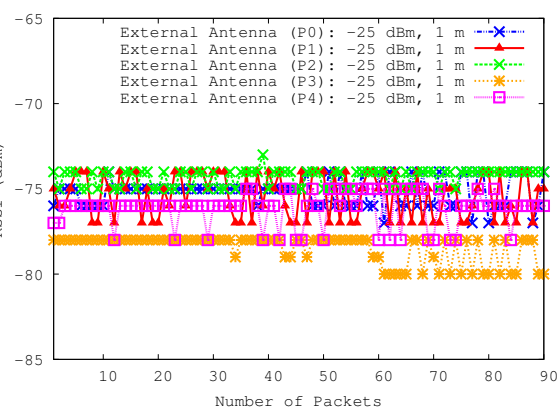

(d)

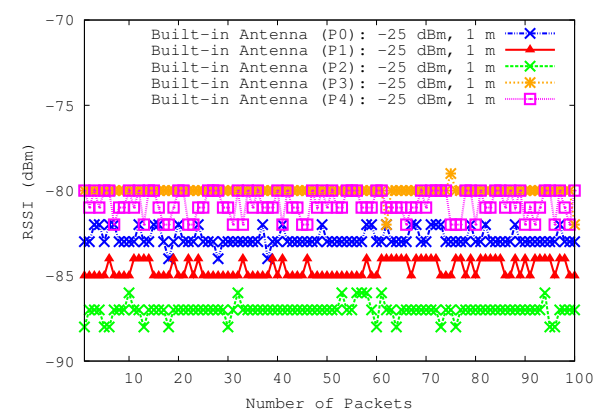

(e)

Figure 11: Number of Packets and RSSI in Anechoic chamber environment. (a) $0 \mathrm{dBm}$ Power. (b) $-10 \mathrm{dBm}$ Power. (c) $-25 \mathrm{dBm}$ Power. (d) External antenna placed at five different locations with $-25 \mathrm{dBm}$ Power. (e) Built-in antenna placed at five different locations with -25 dBm Power 


\subsection{Outdoor Garden Environment}

In outdoor garden environment, we conduct experiments in the absence of any reflecting material except the ground of the garden.

We compare the RSSI values of built-in antenna and external IFA antenna in Outdoor Garden Environment, for each received packet, with 0 dbm, -10 $\mathrm{dBm}$ and $-25 \mathrm{dBm}$ power levels, as can be seen in Fig. 12(a), 12(b) and 12(c). More precisely, results in Fig. 12(a), 12(b) and 12(c) were taken when the nodes were place 1 meter apart. While Fig. 12(d) and 12(e) shows the results when the nodes were 1 meter apart and the sender node is placed at five different locations (cf. Fig. 5).

In the outdoor garden environment, the IFA antenna provides higher power levels i.e., RSSI values at the receiver. More precisely, when power level is 0 $\mathrm{dBm}$, the external IFA antenna provides $-47 \mathrm{dBm}$ power compared to $-62 \mathrm{dBm}$ power for the built-in antenna, i.e., the gain of $15 \mathrm{~dB}$. When power level is -10 $\mathrm{dBm}$, the external IFA antenna provides $-57 \mathrm{dBm}$ power compared to $-73.0 \mathrm{dBm}$ power for the built-in antenna, i.e., the gain of $16 \mathrm{~dB}$. And when the power level is $-25 \mathrm{dBm}$, the external IFA antenna provides $-76 \mathrm{dBm}$ power compared to -83 $\mathrm{dBm}$ power for the built-in antenna, i.e., the gain of around $7 \mathrm{~dB}$.

We then perform an statistical analysis of the received power for both the built-in and external antenna in the outdoor garden environment. The graphical representation in Fig. 13 permits to know the probability of the received power level during the measurement campaign. As mentioned earlier, for the same probability the received signal can be higher or lower according to the antenna and this is much more useful to compare the antennas than by just looking at the time domain received power. Thus, we plot Empirical Cumulative Distribution Function (ECDF) on the log scale [42] that we obtained in the outdoor garden environment. Fig. 13 shows the empirical cumulative distribution function for the two antennas. At $10 \%$ of reliability, Fig. 13 shows the gain of $7 \mathrm{~dB}$ improvement with external antenna in all the cases. This validates the effectiveness of the external antenna. 


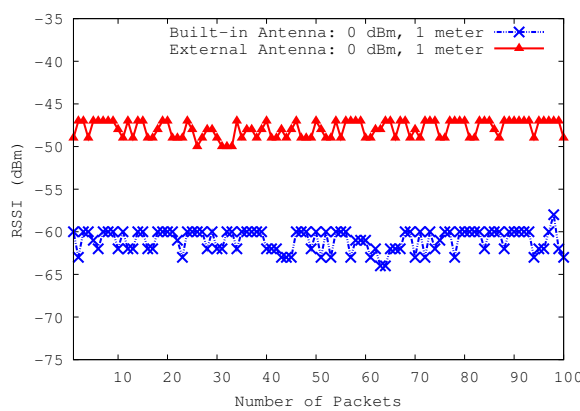

(a)

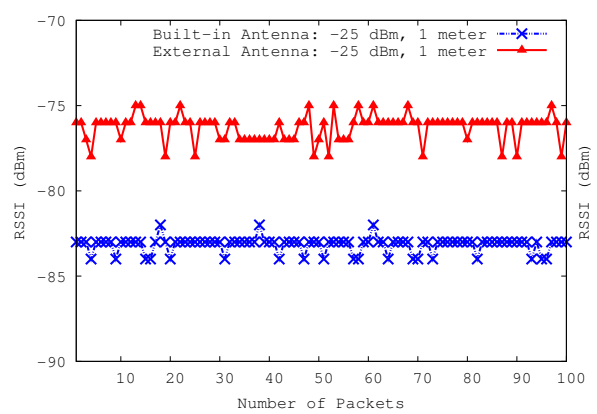

(c)

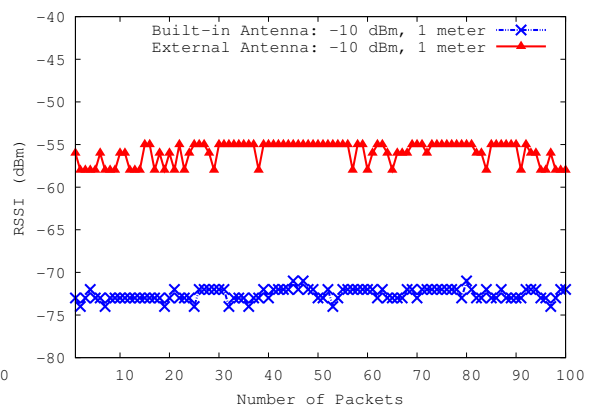

(b)

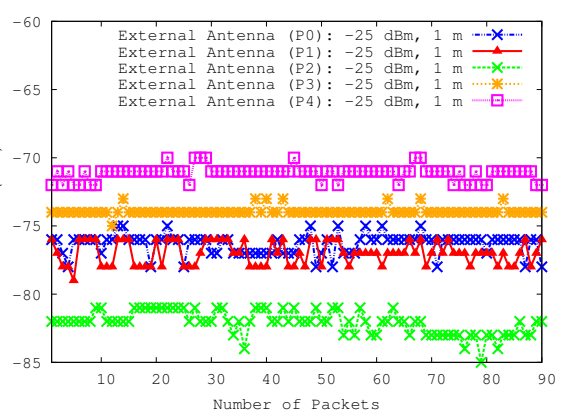

(d)

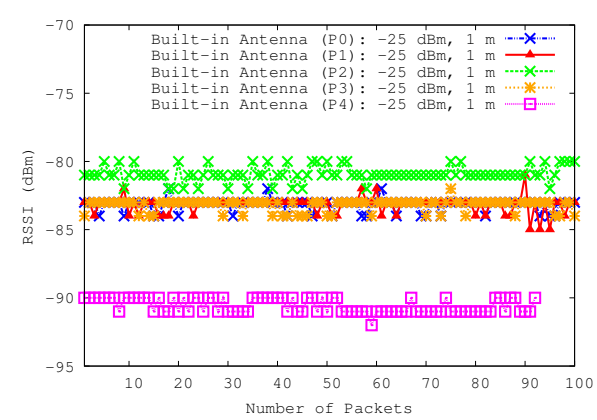

(e)

Figure 12: Number of Packets and RSSI in Outdoor Garden environment. (a) 0 dBm Power. (b) $-10 \mathrm{dBm}$ Power. (c) $-25 \mathrm{dBm}$ Power. (d) External antenna placed at five different locations with $-25 \mathrm{dBm}$ Power. (e) Built-in antenna placed at five different locations with $-25 \mathrm{dBm}$ Power. 


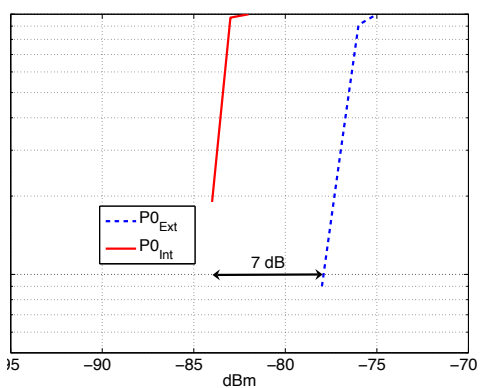

(a)

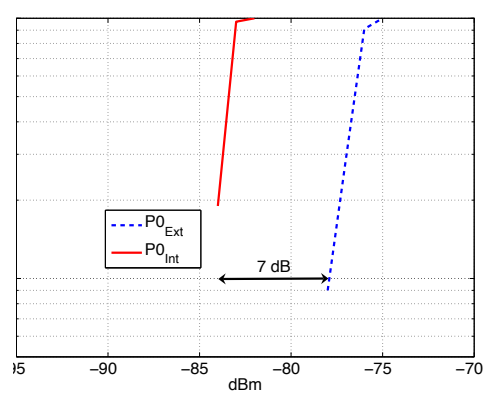

(c)

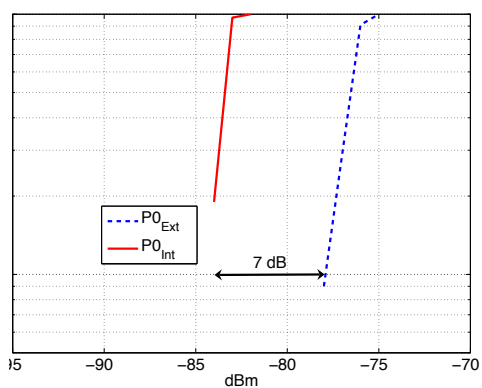

(b)

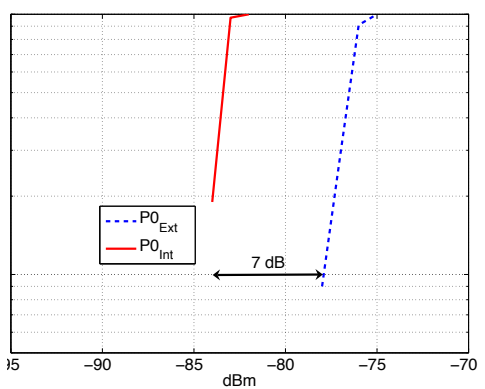

(d)

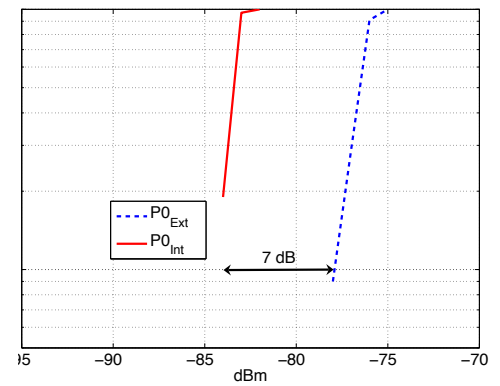

(e)

Figure 13: Emperical Cumulative Distributed Function for External and Built-in Antenna in the Outside Garden Environment (a) position 0 (b) position 1 (c) position 2 (d) position 3 (e) position 4 


\subsection{Concluding Remarks}

In this section, we present our concluding remarks for the extensive performance analysis results. Results in section 3.1, 3.2, 3.3 and 3.4 shows that when dealing with different environments (office environment, outdoor garden environment, reverberation chamber, and anechoic chamber), we observed fluctuation on RSSI values for both antennas. This provide us the basis and motivate us to exploit antenna diversity. Results also confirm that if external antenna is used, the gain of RSSI is significant.

In this paper, we argued that antenna diversity can be used to achieve gain in RSSI. We also noticed that spatio-temporal fluctuations or interference may lead to the degradation of signal power (RSSI). We showed that this happens very frequently and if a proper strategy is designed to change the intelligent antenna selection decision adaptively, we can achieve higher gain.

In the next section, we discuss our proposed EWMA based adaptive intelligent antenna selection decision strategy.

\section{EWMA based Intelligent Antenna Selection Strategy}

EWMA is a one-step prediction technique developed by [27], which has been widely used in different applications, such as anomaly detection [28], bandwidth prediction etc. In EWMA, higher importance is given to the more recent observations. Thus, EWMA is very suitable for predicting the RSSI value on a particular antenna. In fact, our goal is to detect any small variation in the RSSI value through EWMA prediction technique and then use the appropriate antenna.

In order to achieve this goal, we enable the wireless sensor nodes to keep the record of the RSSI values and this record will be served as an input to predict the RSSI value. Initially, no prediction will be done and each wireless sensor node just collect the historical data of RSSI values on different antennas. Once the nodes have some historical data, the receiving node will start predicting the RSSI values for both antennas. The antenna which provides strong predicted 


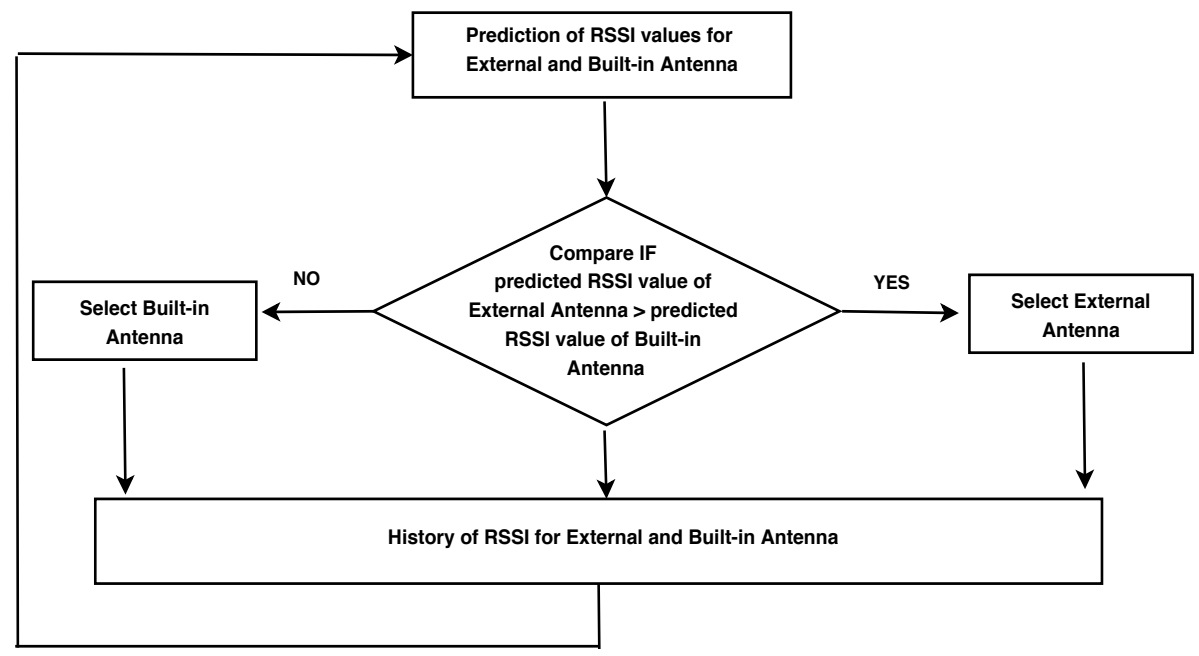

Figure 14: Flow chart shows the decision making by the sensor nodes to select the antenna.

RSSI value at time instant $t$ will then be selected for the next reception period, thus achieving the goal of antenna diversity. Fig. 14 shows the decision making by the sensor nodes to select the appropriate antenna according to the varying RSSI value. As can be seen in Fig. 14, first sensor nodes predict the values of RSSI for both built-in and external antenna. If the RSSI value of external antenna is greater than the built-in antenna, sensor node will switch to the external antenna, else vice versa. Then, sensor nodes keep these observed values of RSSI in the memory. Finally, these stored RSSI values will be then feedback to predict for the next time.

Let $\triangle R S S I(t)^{E}$ be the mean of historical values of RSSI, $\triangle R S S I$ (t) be the observation at time $\mathrm{t}$ and $\gamma$ is a smoothing factor $(0 \leq \gamma \leq 1)$. The RSSI value at time $(t+1)$ i.e., $\triangle R S S I(t+1)$ can be calculated as:

$$
\triangle R S S I(t+1)^{E}=(1-\gamma) \triangle R S S I(t)^{E}+\gamma \triangle R S S I(t)
$$

In this manner, the wireless sensor node can predict the value of RSSI at time instant $t$ and make an intelligent antenna selection decision. This decision is achieved by implementing an adaptive electronic switch which reacts on the predicted value of RSSI. 


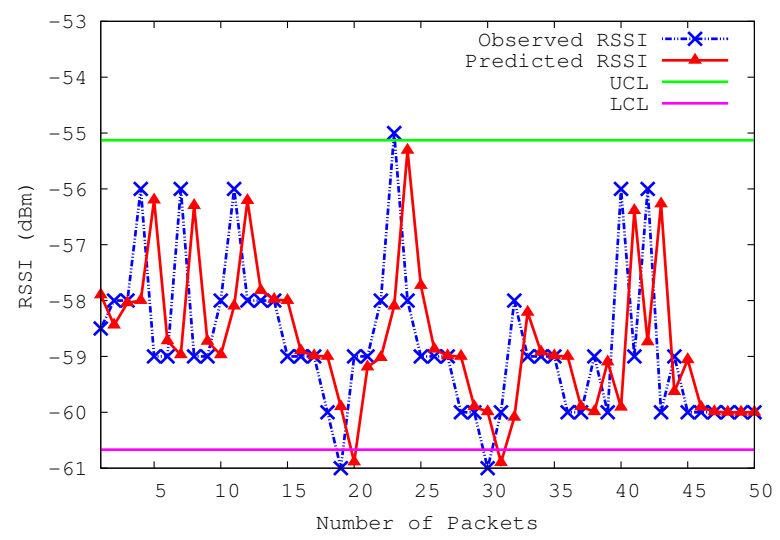

Figure 15: Observed and predicted values of RSSI for the external antenna, when nodes are 1 feet apart at $-25 \mathrm{dBm}$.

However, it may be possible that due to some abrupt fluctuation in the environment, the RSSI value vary a lot from the mean value of RSSI. This feature is exploited for intrusion detection [18] and for monitoring the human activity. In this paper, we focus on the consistent values of RSSI, instead of identifying the anomaly. In case, any anomaly is detected, the wireless sensor nodes will not take into account those anomalies. In order to identify any anomaly in $\triangle R S S I(\mathrm{t})$, we propose to use two control limits, Upper Control Limit (UCL) and Lower Control Limit (LCL), which can be calculated as:

$$
\begin{gathered}
U C L_{\triangle R S S I}=\triangle R S S I(t)+k s \triangle R S S I \\
L C L_{\triangle R S S I}=\triangle R S S I(t)-k s_{\triangle R S S I} \\
s_{\triangle R S S I}^{2}=\frac{\gamma}{2-\gamma} s^{2}
\end{gathered}
$$

where $s^{2}$ is the variance of the generated and updated database, which is used to calculate UCL and LCL, and $\mathrm{k}$ is set equal to 3 [29].

Fig. 15 shows the observed and predicted values of RSSI for the external antenna, when sensor nodes are 1 feet apart with $-25 \mathrm{dBm}$ transmission power. It can be clearly seen in the figure that EWMA technique predicts very well in accordance with the observed RSSI values, thus showing the effectiveness of the 


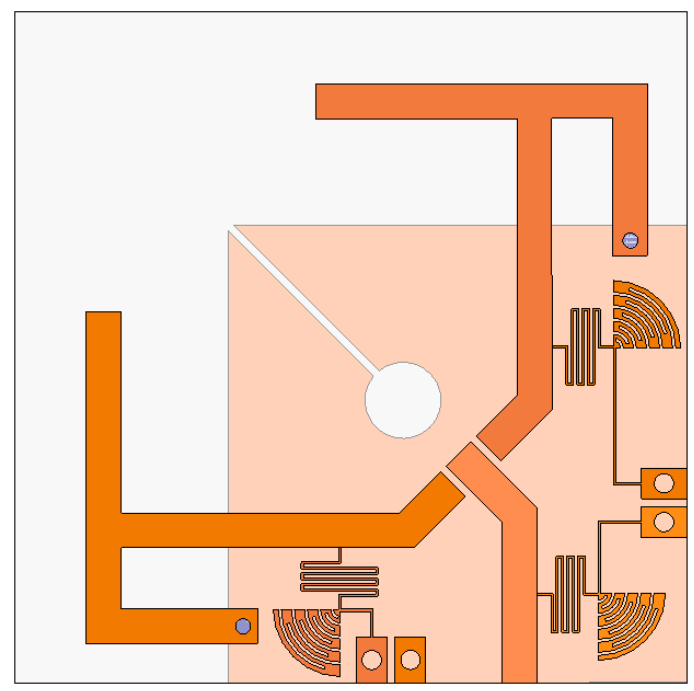

Figure 16: Intelligent Switch Design

prediction technique. In the next seciton, we describe our diversity antenna and intelligent switch design.

\subsection{Diversity Antenna and Intelligent Switch Design}

Fig. 16 shows our diversity antenna design. This diversity antenna is attached with the TelosB mote with the port through an electronic switch. Fig. 17 shows the design of our electronic switch.

Switching between antennas is made by two diodes specially used for this purpose. DC power is applied to the DC1 or DC2 ports to swith ON/OFF one of the diodes. RF/DC power decoupling is achieved with special techniques used in microwave engineering and consists in using printed capacitors and quarter wavelength lines.

The antennas and the switching system is shown on this drawing. By transparency the bottom ground plane is also visible. This one contains a quarter wavelength slot which permits to isolate one antenna from the another at 2.4 GHz. This technique effectively chokes the currents on the ground plane. In this way one antenna is not affected by the other close one. 


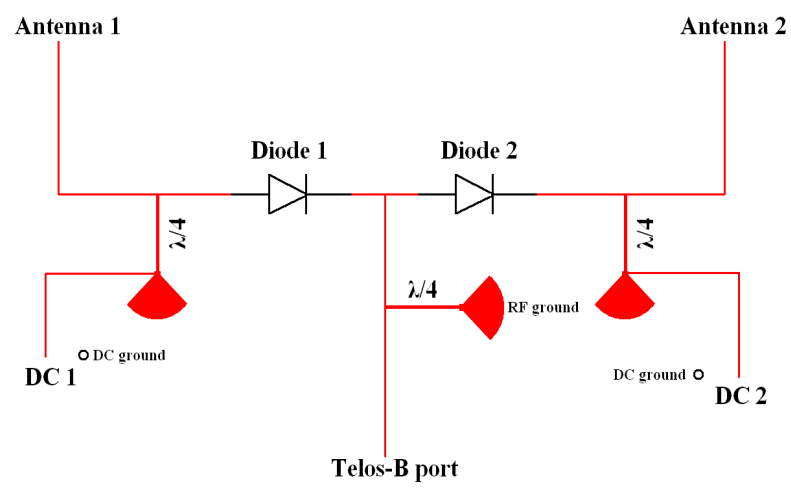

Figure 17: Circuit diagram for intelligent switch design

\section{Open Issues and Challenges}

We now highlight some open issues and challenges related with intelligent antenna selection and antenna diversity in the context of WSNs.

- Antenna switching tradeoff: Antenna switching also incurs with delay but if intelligently done, brings increase in throughput and helps to select reliable channels. Thus, there is need to explore this tradeoff.

- Antenna efficiency and switch energy consumption: Very less work has been done so far in the context of antenna efficiency and switch energy consumption of different antennas. More precisely, antenna efficiency means the energy consumption pattern of wireless sensor nodes antenna, which vary with the design of antenna used. Thus, it is important to study in detail the antenna efficiency.

- Cross-layer information for antenna selection: From the cross-layer perspective, several architectures have been proposed to optimize the PHY layer information to make better decisions on the upper layers [30, 31]. Thus, there is also a need to consider the cross-layer information for the design of antenna selection metric. 
- Local versus Global knowledge of channels: Data dissemination is one of the major applications in WSNs [32]. In these types of applications, there may not exist a centralized entity and the wireless sensor nodes communicate in multi-hop fashion. In addition, wireless sensor nodes are required to rely on locally inferred information for the antenna selection decision. The local and global information regarding the channel conditions and the absence of centralized entity, may have an impact on the overall performance of WSNs, which is also need to be investigated in more detail.

- Communication improvement in Vehicular Sensor Networks: In Vehicular Sensor Networks, antenna diversity can improve the communication in the urban environment where channel state change quickly but there is a need to investigate this issue in detail.

- Use of Antenna diversity for node localization: Antenna diversity in conjunction with RSSI can be helpful to locate the nodes position. As discussed in [33], there is a need to further investigate the affect of antenna diversity along with RSSI, to accurately locate wireless sensor nodes position.

- Direction of arrival estimation in reverberation chamber and real environment: The direction of arrival estimation in reverberation chamber and real environment is really important to understand the behaviour of the deployed wireless sensor network. This will certainly help us to improve the performance of the antennas, as well as the WSN system.

- Impact of packet sending delay: During our experiments, we observed that due to the multipath effect and direction of arrival estimation, some packets were lost in the reverberation chamber. As per our initial understanding, this is due to the very short sending delay between the two consecutive packets. There we need to properly analyze this affect of short and long delay between packet sending and their impact of the delivery ratio, in the reverberation chamber. 


\section{Related Work}

In this section, we start with the applications of RSSI. We then discuss the impact of antenna orientation and antenna diversity, and finally, we discuss the related work on multi-radio wireless sensor networks.

Applications of RSSI: RSSI is very sensitive to the environment and vary even with a very small flucutation of the RF signal. Due to this sensitive nature of RSSI, it has been widely used in the literature for different applications. For instance, RSSI has been used for intrusion detection [18]. In [18], the authors observed the RSSI values of the TelosB motes to detect the human activity. Localization of objects through RSSI is another application area, where lot of work has been done. The effects of fast moving objects, such as cars, on RSSI in wireless sensor networks is studied in [19]. The effect of RSSI on fast moving rotating structures is done in [20]. In [20], the authors mounted the wireless sensor nodes inside the computer numerical control lathe machine and studied the effects of rotating wireless sensor nodes on signal performance.

In [34], the authors developed a tool for wireless sensor networks for node localization through RSSI. A ZigBee based wireless sensor network localization for cattle monitoring in grazing fields is done in [35]. An experimental comparison of RSSI-based localization algorithms for indoor wireless sensor networks is done in [36].

Impact of Antenna Orientation and Antenna Diversity: The impact of antenna orientation on the performance of wireless sensor networks is done in [21]. They showed that antenna orientation has a significant impact on RSSI. The authors then proposed a routing protocol, which is based on antenna orientation. A modified model of external antenna is discussed in [22] for WSNs but the authors concentrate on range improvement with the help of improved antenna impedance matching. The application of antenna diversity in wireless sensor networks for animal monitoring is presented in [6].

In [37], the authors presented MoteFinder and discuss the use of two simple objects to increase the directivity of antennas in wireless sensor networks. The 
authors considered simpler tinfoil cylinder and a cantenna and studied the impact of them on the radio propagation of a mote. The direction of arrival estimation in reverberation chamber with the help of wireless sensor networks is done in [38]. The authors in [39] proposed a directional-antenna-assisted reactive routing protocol, Interest Dissemination with Directional Antennas (IDDA). The authors in [41] addressed the problem of medium access control in adhoc networks with fully adaptive array antennas but the authors focused on CSMA/CA. A very nice discussion on directional antennas is done in [40] but they didn't focus on wireless sensor networks.

Multi-radio Wireless Sensor Networks: The authors in [23, 24, 25] concentrate on multi-radio wireless sensor networks, where two separate radio modules were attached with the sensor nodes, thus increasing the deployment cost of WSNs and requires bigger and more complex wireless sensor devices. While, in this paper, we focus on single CC2420 built-in radio chip, with one external antenna (compatible with CC2420 built-in chip, thus omitting the need to add any additional radio chip) to exploit antenna diversity for making intelligent antenna selection decision.

\section{Conclusion and Future Work}

In this paper, we discussed our results of the newly deigned radio module (Inverted F Antenna) for $2.4 \mathrm{GHz}$ wireless sensor networks. We first performed extensive experiments to understand the behaviour of RSSI values for the builtin and external antenna, in both lab and outdoor environments. We then proposed an EWMA based intelligent antenna selection strategy for wireless sensor nodes. We also provided the prototype of diversity antenna as well as the circuit diagram of intelligent antenna switch. Experimental results confirmed the effectiveness of our proposed radio module over the built-in TelosB radio module.

There are a number of open problems related to the design of intelligent antenna selection for WSNs. First, we plan to study different routing metrics with antenna diversity under multi-hop network configuration. Second, we want 
to study the efficiency of IFA antenna. We also want to exploit the issues and challenges discussed in section 5. Third, we want to perform detail studies on how the antenna selection improves a WSN's performance in terms of packet delivery rate, throughput, latency, energy efficiency, etc. Fourth, we also intend to study how multi-channel communication can also help to improve the WSN performance and to study the cases where multi-channel communication is advantageous and cases where multiple antennas are better. Finally, our goal is to integrate software defined switching capability to TelosB motes to change the antenna decision adaptively. This antenna switching decision will be based on link quality metrics, i.e., RSSI.

\section{References}

[1] Th. Arampatzis, J. Lygeros, S. Manesis, A Survey of Applications of Wireless Sensors and Wireless Sensor Networks, Proceedings of the 2005 IEEE MED Conference, pp.719-724, 27-29 June 2005.

[2] Yun Wang, Peizhong Shi,Kai Li and Zhikui Chen, An energy efficient medium access control protocol for target tracking based on dynamic convey tree collaboration in wireless sensor networks, International Journal of Communication Systems, 2012; 25:1139-1159.

[3] M. Radenkovic, and B. Wietrzyk, Wireless Mobile Ad-hoc Sensor Networks for Very Large Scale Cattle Monitoring, in Proceedings of ASWN'06, Berlin, Germany, 2006.

[4] Y. Guo, P. Corke, G. Poulton, T. Wark, G. Bishop-Hurley, D. Swain, Animal Behaviour Understanding using Wireless Sensor Networks, 31st IEEE Conference on Local Computer Networks, pp.607-614, 14-16 Nov. 2006.

[5] Kwong Kae Hsiang, K. Sasloglou, H. G. Goh, Tsung Ta Wu, B. Stephen, M. Gilroy, C. Tachtatzis, I. A. Glover, C. Michie, I. Andonovic, Adaptation of wireless sensor network for farming industries, Sixth International Conference on Networked Sensing Systems (INSS), pp.1-4, 17-19 June 2009. 
[6] Konstantinos Sasloglou, Ian A. Glover, Hock Guan Goh, Kae Hsiang Kwong, Michael P. Gilroy, Christos Tachtatzis, W. Craig Michie, Ivan Andonovic, Antenna and Base-Station Diversity for WSN Livestock Monitoring, Wireless Sensor Network Journal, volume 1, no. 5, pages 383-396, 2009.

[7] AVR2023 - AT86RF231 PCB reference design for antenna diversity, www.atmel.com

[8] T. S. Rappaport, Wireless Communication Principles and Practice, 2nd Edition, Prentice Hall, New Jersey, 1996.

[9] Carles Gomez, Antoni Boix, and Josep Paradells. Impact of lqi-based routing metrics on the performance of a one-to-one routing protocol for IEEE 802.15.4 multihop networks. EURASIP Journal on Wireless Communications and Networking, 2010:6:1-6:20, July 2010.

[10] Kannan Srinivasan and Philip Levis. RSSI is under appreciated. In Proceedings of EmNets, 2006.

[11] Ardalan Amiri Sani, Lin Zhong, and Ashutosh Sabharwal, Directional Antenna Diversity for Mobile Devices: Characterizations and Solutions, MobiCom 2010.

[12] Kai Han, Liu Xiang, Jun Luo, and Yang Liu, Minimum-Energy Connected Coverage in Wireless Sensor Networks with Omni-Directional and Directional Features, MobiHoc 2012.

[13] Mubashir Husain Rehmani, Thierry Alves, Stephane Lohier, Abderrezak Rachedi, Benoit Poussot, Towards Intelligent Antenna Selection in IEEE 802.15.4 Wireless Sensor Networks, ACM MobiHoc'12 (Poster session), South Carolina, USA, June 11-14, 2012.

[14] Mubashir Husain Rehmani, Abderrezak Rachedi, Stephane Lohier, Thierry Alves, Benoit Poussot, On the Feasibility of making Intelligent Antenna Selection Decision in IEEE 802.15.4 Wireless Sensor Networks, ComComAp'13, Hong Kong, China, 2-4 April 2013. 
[15] Moteiv Corporation, telos datasheet http://www2.ece.ohiostate.edu/ bibyk/ee582/telosMote.pdf

[16] MEMSIC Inc, http://www.memsic.com, 2011.

[17] Chipcon cc2420 datasheet, http://www.chipcon.com, 2011.

[18] Pius W.W. Lee, Winston K.G. Seah, Hwee-Pink an, and Zexi Yao, Wireless Sensing Without Sensors - An Experimental Approach, IEEE PIMRC 2009.

[19] Syed Hassan Ahmed, Safdar Hussain Bouk, Amjad Mehmood, Nadeem Javaid and Iwao Sasase, Effect of Fast Moving Object on RSSI in WSN: An Experimental Approach, http://arxiv.org/abs/1202.4137, 2012.

[20] Kuang-Ching Wang,Lei Tang, and Yong Huang, Wireless sensors on rotating structures: performance evaluation and radio link characterization, WinTECH 2007.

[21] M. Wadhwa, Min Song, Vinay Rali, and Sachin Shetty, The impact of antenna orientation on wireless sensor network performance, IEEE ICCSIT 2009.

[22] Johnston, R.H.; Finvers, I.G.; Haslett, J.W.; , Antenna optimization for RF mote communications, IEEE Antennas and Propagation Society International Symposium 2006, July 2006

[23] Jeremy Gummeson, Deepak Ganesan, Mark D. Corner, and Prashant Shenoy, An Adaptive Link Layer for Range Diversity in Multi-radio Mobile Sensor Networks, IEEE INFOCOM 2009.

[24] Dimitrios Lymberopoulos, Nissanka B. Priyantha, Michel Goraczko and Feng Zhao, Towards Energy Efficient Design of Multi-Radio Platforms for Wireless Sensor Networks, International Conference on Information Processing in Sensor Networks, 2008. 
[25] Raja Jurdak, Kevin Klues, Brano Kusy, Christian Richter, Koen Langendoen, and Michael Brunig, Opal: A Multiradio Platform for High Throughput Wireless Sensor Networks, IEEE Embedded Systems Letters 3(4): 121124 (2011).

[26] Kent Rosengren and Per-Simon Kildal, Study of Distributions of Modes and Plane waves in Reverberation chambers for the characterizaiton fo antennas in a Multi-path environment, Microwave and Optical Technology Letters, Vol. 30, No. 6, September 20, 2001.

[27] S. W. Roberts, Control Chart Test Based on Geometric Moving Average, Technometrics, Vol. 42, No. 1, pp. 97-101,Feb 2000.

[28] Ye Nong, S. Vilbert, and Chen Qiang, Computer intrusion detection through EWMA for autocorrelated and uncorrelated data, IEEE Transactions on Reliability, vol.52, no.1, pp. 75- 82, March 2003.

[29] J. M. Lucas and M. S. Saccucci. Ewma Control Schemes: Properties and Enhancements. Technometrics, 32, Feb 1990.

[30] Lucas D.P. Mendes, Joel J.P.C. Rodrigues, A survey on cross-layer solutions for wireless sensor networks, Elsevier JNCA, 2010.

[31] Qiong Shi, Cristina Comaniciu, Dandan Wang and Ufuk Tureli, Crosslayer MAC design for location-aware wireless sensor networks, International Journal of Communication Systems, 2011; 24:872-888.

[32] Mubashir Husain Rehmani and Aline Carneiro Viana (Advisor), Decoupling Data Dissemination from the Mobile Sink Trajectory in Wireless Sensor Networks: Current Research and Open Issues, Masters Thesis, Laboratory of Signals and Systems, Supelec and University of Paris Sud-11, July 2008.

[33] Wei Lifeng Wei, Liu Na, Pang Yujun, Wang Qinghui, Liu Xiaomei, Application of antenna diversity technique on Wireless Sensor Network localization, CCDC, 2010, pp.1412-1416, 26-28 May 2010. 
[34] Nick Verbaendert, David Henderickx and Jeroen Doggen, Emote: A Wireless Sensor Network Monitoring Tool With Node Localization Using RSSI, PAPERS OF THE E-LAB MASTER THESES 2007-2008.

[35] Juan Ignacio Huircana, Carlos Munoza, Hector Younga, Ludwig Von Dossowa, Jaime Bustosa, Gabriel Vivallob, Marcelo Toneattib, ZigBee-based wireless sensor network localization for cattle monitoring in grazing fields, Elsevier Computers and Electronics in Agriculture Journal, 2010.

[36] Giovanni Zanca, Francesco Zorzi, Andrea Zanella and Michele Zorzi, Experimental comparison of RSSI-based localization algorithms for indoor wireless sensor networks, REALWSN'08, April 1, 2008, Glasgow, United Kingdom.

[37] Olga Saukh, Robert Sauter, Jonas Meyer, Pedro Jose Marron, MoteFinder: A Deployment Tool For Sensor Networks, REALWSN'08, April 1, 2008, Glasgow, United Kingdom.

[38] Murat Arabaci and Robin N. Strickland, Direction of Arrival Estimation in Reverberant Rooms Using a Resource-Constrained Wireless Sensor Network, IEEE International Conference on Pervasive Services, July 2007.

[39] Y. Wu, Y., L. Zhang, Y. Wu, 2. Niu, Motion-indicated interest dissemination with directional antennas for wireless sensor networks with mobile sinks, IEEE Transactions on VT, Volume 58 (2), 2009, pp 977-989 .

[40] R. Ramanathan, J. Redi, C. Santivanez, D. Wiggins, S. Polit, Ad hoc networking with directional antennas: a complete system solution, IEEE JSAC, vol.23 (3), pp. 496- 506, March 2005.

[41] Karthikeyan Sundaresan and Raghupathy Sivakumar, On the medium access control problem in Ad hoc networks with smart antennas, MobiHoc Poster, Volume 7 Issue 3, July 2003, pages 25 - 26.

[42] Fontan Perez F., Espineira Marino P., Modeling the Wireless Propagation Channel, Wiley edition 2008, ISBN 978-0-470-72785-0 